EPJ Web of Conferences 73, 08005 (2014)

DOI: $10.1051 /$ epjconf/20147308005

(C) Owned by the authors, published by EDP Sciences, 2014

\title{
The Large Hadron electron Collider at CERN
}

Alessandro Polini ${ }^{\mathrm{a}}$ on behalf of the LHEC Collaboration

INFN Bologna, via Irnerio 46, Bologna, Italy

\begin{abstract}
The Large Hadron electron Collider ( $\mathrm{LHeC})$ is a proposed facility which will exploit the new world of energy and intensity offered by the LHC through collisions with a new $60 \mathrm{GeV}$ electron beam. Designed for synchronous operation with the other LHC experiments, the $\mathrm{LHeC}$ will be a high luminosity $e p$ and $e A$ collider with a wide ranging physics program on high precision deep inelastic scattering and new physics. Highlights from the physics program will be illustrated along with details from the accelerator, interaction region and detector design.
\end{abstract}

\section{Introduction}

Deep inelastic lepton-hadron scattering is the cleanest and most precise probe of parton dynamics in protons and nuclei. By adding a $60 \mathrm{GeV}$ electron beam to the $\mathrm{LHC}$, the $\mathrm{LHeC}$ represents the only short term proposal for $\mathrm{TeV}$-scale lepton-hadron scattering ideally complementing the LHC $p p, A A$ and $p A$ program and its discovery potential for physics beyond the Standard Model with high precision deep inelastic scattering (DIS) measurements.

This writeup summarizes some of the central aspects of the LHeC project including a selection of physics highlights and an overview of the accelerator and the detector design. Latest updates and a project roadmap are also presented. More details can be found in the extensive conceptual design report (CDR) [1] and in the references given below.

\section{The accelerator}

For the electron acceleration the CDR described two options for the $\mathrm{LHeC}$ accelerator complex: a ringring (RR) and a linac-ring (LR) configuration. More recently it was decided to pursue the technical design work for the LR configuration only, mainly for its lower impact on the LHC program, keeping the $\mathrm{RR}$ as a backup. The default electron beam energy is set to $60 \mathrm{GeV}$ with the luminosity being constrained by a chosen wall-plug power limit of $100 \mathrm{MW}$ for the lepton beam. The layout of the electron accelerator is shown in Fig. 1: it comprises two linear sections, each $1 \mathrm{~km}$ in length and with a modest acceleration gradient of $20 \mathrm{MeV} \mathrm{m}^{-1}$, linked by two arcs into a racetrack configuration with a circumference almost $9 \mathrm{~km}$ long. Electrons circulate in this machine three times before being delivered tangentially to an

\footnotetext{
ae-mail: alessandro.polini@bo.infn.it
}

This is an Open Access article distributed under the terms of the Creative Commons Attribution License 4.0, which permits unrestricted use, distribution, and reproduction in any medium, provided the original work is properly cited. 


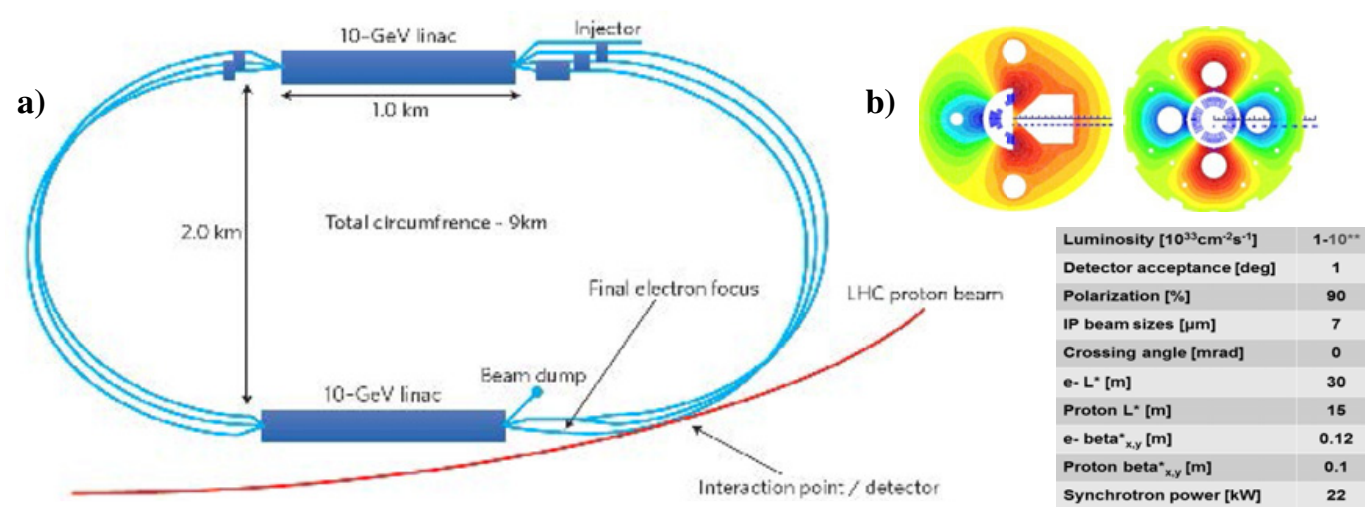

Figure 1. a) The proposed layout of the LHeC. An electron accelerator, comprising two linear accelerators in racetrack configuration, could deliver an intense $60-\mathrm{GeV}$ beam to an interaction point with the existing LHC, and generate electron-proton collisions at a centre-of-mass energy of $1.3 \mathrm{TeV}$. Some of the accelerator parameters are listed in the table on the left. b) An illustration of the Q1 and Q2 magnets of the inner triplet which include the field free region for the spectator beam and the aperture for the synchrotron fan generated by the steering of the electron beam.

interaction point in the LHC, and colliding with the LHC beam at an electron-proton centre-of-mass energy of $1.3 \mathrm{TeV}$. The electron beam then decelerates through the same racetrack structure, allowing most of the energy to be recovered. The $\mathrm{LHeC}$ operation is foreseen in parallel with the high-luminosity phase of the LHC, without disrupting data-taking by the other experiments in the LHC ring. The design luminosity of $10^{33} \mathrm{~cm}^{-2} \mathrm{~s}^{-1}$ is already an improvement by around two orders of magnitude over that reached at HERA. More recent estimates [4] based on the LHC performance during Run-1 and on further optimizations, indicate that luminosities of order of $10^{34} \mathrm{~cm}^{-2} \mathrm{~s}^{-1}$ are achievable.

\section{Deep inelastic scattering at the LHeC}

The $\mathrm{LHeC}$ physics spectrum is very wide and unique[1-4]; here, for space limitations, only a quick overview can be given. The $\mathrm{LHeC}$ physics is well summarized by Fig. 2 a which shows the available kinematic range as a function of Bjorken- $x$, the momentum-fraction carried by the struck parton in the proton, and $Q^{2}$, the lepton four-momentum transfer squared. The coverage of previous DIS experiments and a map of the relevant physics measurements are also indicated in the figure. The LHeC extends by a factor of 20 the kinematic range at low- $x$ down to $10^{-6}$ and at high- $Q^{2}$ making a complete unfolding of the proton flavour structure possible for the first time and providing an order of magnitude improvement in experimental precision on the strong coupling constant $\alpha_{S}$. This is particularly relevant as these elements and their uncertainties enter in any process inspected at the LHC.

Figure $2 b$ illustrates the potential improvement in the gluon PDF uncertainty over the full range of $x$ from $\mathrm{LHeC}$ data by comparing the uncertainties from a fit to just HERA-I combined data and to a fit to these data plus LHeC pseudo-data. Fits to HERA plus BCDMS fixed target data and HERA plus LHC $W$-asymmetry data are also illustrated but these do not bring such a dramatic reduction in uncertainty, even when current LHC data have their uncertainties reduced to reflect our best estimate of the ultimate achievable accuracy.

The improvement in the knowledge of the parton densities at high- $x$ is crucial for searches of new states and physics beyond the standard model. As an example, the dominant SUSY production channels at the LHC are assumed to be squark-(anti)squark, squark-gluino, and gluino-gluino pair production. 

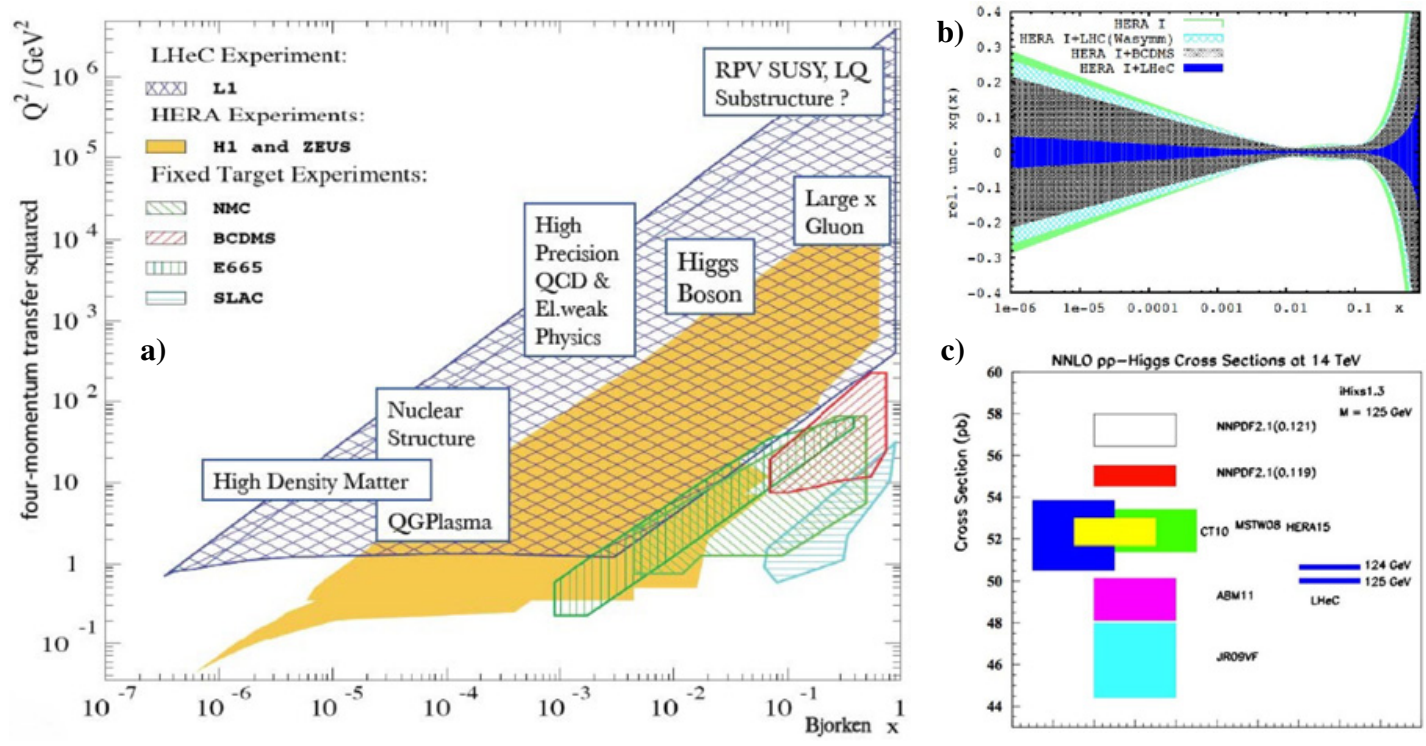

Figure 2. a) The kinematic plane in Bjorken- $x$ and resolving power, $Q^{2}$, showing the coverage of fixed-target experiments, HERA and the LHeC. The mapping of the relevant physics program onto the plane is also indicated. b) The PDF uncertainty on the gluon distribution from a fit to just HERA-I data, HERA-I+BCDMS data, HERAI+LHC W-asymmetry data and HERA-I+LHeC pseudo-data corresponding to $50 \mathrm{fb}^{-1}$. c) NNLO calculation of the Higgs production cross section in $p p$ scattering at the design LHC energy using the iHixs program. The bands on the left side represent the uncertainties of various PDF sets. The full experimental uncertainty estimated with the $\mathrm{LHeC} \mathrm{PDFs}$ for comparison is shown on the right. The $\mathrm{LHeC}$ would provide the means to derive Higgs mass values from LHC cross section measurements.

If gluinos of $2 \sim 4 \mathrm{TeV}$ mass do exist, their discovery and kinematic characterisation will depend on the capability to predict their production cross section with good precision.

At low- $x$, as predicted theoretically and confirmed at HERA, the proton structure is characterized by a rapid increase in the density of partons. It is a natural question as to whether this rise continues to indefinitely small $\mathrm{x}$, or whether, like the $W W$ scattering cross-section, there is a mechanism to tame the growth and satisfy unitarity constraints. One proposed mechanism is the recombination of pairs of gluons when they are packed at a sufficient density to saturate. This behaviour of the densely packed gluons in the low- $x$ regime of quantum chromodynamics may be related to the confinement of quarks and the generation of most of the masses of hadrons.

The extension in kinematic range over previous DIS experiments becomes even larger when colliding electrons with heavy nuclei. Four orders of magnitude in $x$ are gained resulting in a unique capability for pinning down saturation phenomena and measuring with unprecedented accuracy the nuclear structure.

Higgs bosons at the LHeC are produced dominantly through vector boson fusion $\left(W^{+} W^{-} \rightarrow H\right.$ or $Z^{0} Z^{0} \rightarrow H$ ). These processes can be cleanly separated, providing high sensitivity to the $W W H$ and $Z Z H$ couplings, which are central to electroweak symmetry breaking and may receive sizeable contributions from physics beyond the standard model. The Higgs may also be reconstructed in its dominant decay mode to pairs of bottom quarks, which is problematic at the LHC. If high enough luminosities are achieved, decays to other final states - such as pairs of charm quarks, or pairs of muons or taus - might also be accessible while the precision gained on $\alpha_{s}$ and the PDFs would allow precise $p p \rightarrow H$ cross section determination at the LHC. Figure $2 \mathrm{c}$ shows the cross section and uncertainties 
as obtained by the inclusive Higgs cross section program iHixs using as input different PDFs sets and the improvement from $\mathrm{LHeC}[4]$.

Finally the $\mathrm{LHeC}$ will also allow improved precision in the determination of electroweak parameters if the electron beams are polarised allowing a simultaneous fit of PDF and EW parameters.

\section{Interaction region and detector design concept}

The $\mathrm{LHeC}$ is the second electron-hadron collider following HERA. Its physics program demands a very high level of precision, as for the measurement of the strong coupling constant $\alpha_{s}$ to per mill uncertainty, and it requires the reconstruction of complex final states, as appear in single top events or charged current Higgs production with subsequent decay into $b b$ final states. The detector acceptance has to extend as close as possible to the beam axis because of the interest in the physics at small and large $x$. The LHeC interaction region poses new constraints coming from a complex optics which includes 3 beams (the interacting protons and electrons and the second spectator proton beam). This is particularly important in the design of the inner triplet magnets (Fig. 1b). In addition, for the baseline LR configuration, a dipole field $(0.15 \sim 0.3 \mathrm{~T})$ along the whole length of the main detector is required to steer the electron beam for head-on collisions and avoid parasitic interactions of the neighboring bunches which at the LHC are $25 \mathrm{~ns}$ apart. The choice of components for the LHeC detector can rely on the experience obtained at HERA, at the LHC, including its detector upgrades currently being developed, and also on detector studies for the ILC. The detector development, while requiring prototyping, may therefore proceed without an extended R\&D program, which fits well with the LHeC being ready for the LHC Phase-II running (around year 2023).

\section{The baseline detector}

The LHeC baseline detector [1] is shown in Fig. 3a. The detector has to be hermetic in order to maximize coverage especially in the forward and backward regions and provide precise energy and missing energy measurements, the latter being the signature for charge-current processes where the incoming electron converts into an outgoing neutrino. The $\mathrm{LHeC}$ detector is asymmetric in design, reflecting the beam energy asymmetry. Moving from the interaction region outwards, a light beampipe (Fig. 3b) surrounded by a precision silicon tracking detector with extended forward and backward parts is required before reaching the electromagnetic calorimetry presently considered to be based on liquid argon technology. A strong solenoidal magnetic field (3.5 Tesla) is needed for momenta separation and long dipoles are required along the whole interaction region $(-14 \mathrm{~m}<z<+14 \mathrm{~m}$, Fig. 3c) providing a field in a region not too extended in radius. The requirement of a precise electron energy measurement and not too large beam-steering magnets, suggest to have the solenoid and the dipoles integrated in a single structure placed immediately outside of the electromagnetic calorimetry. The hadronic calorimeter (iron-scintillator) surrounds therefore the magnet system and is enclosed in a muon tracker system. In the endcaps, dedicated calorimeters are foreseen to precisely measure forward high energy products (silicon-tungsten) or the scattered electron (silicon-copper). The inner detector dimensions along the beamline are constrained by the radial extension of the beam pipe in combination with maximum polar angle coverage $\left(1^{\circ}\right.$ and $\left.179^{\circ}\right)$ for forward going final state particles and backward scattered electrons at low $Q^{2}$, respectively. The outer radial size is mainly determined by the requirement of full energy containment of hadronic showers in the calorimeter. The main detector is complemented by hadron tagging detectors (not shown) in the forward direction and a polarimeter and luminosity measurement system backwards. 


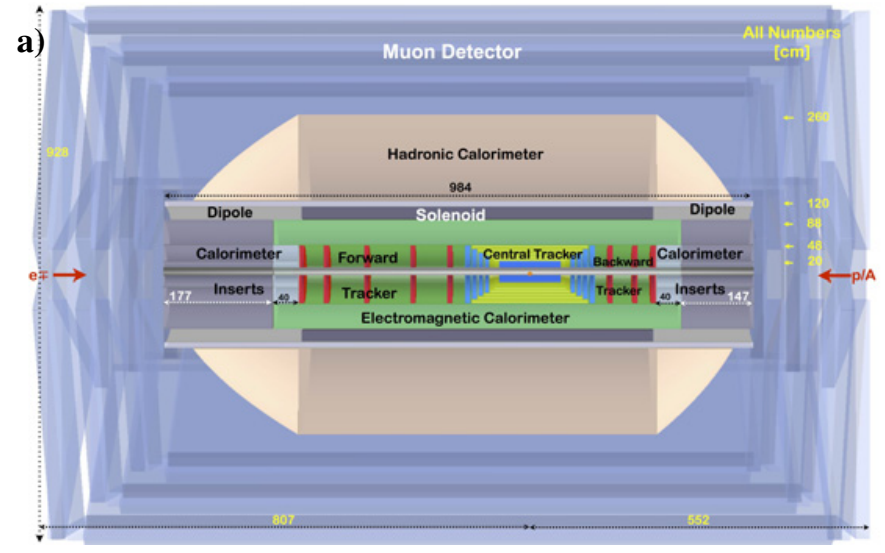

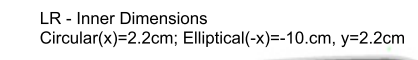

b)

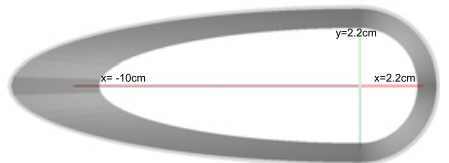

c)

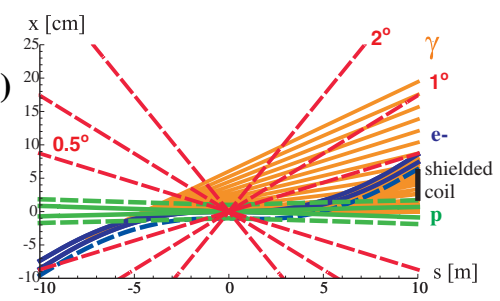

Figure 3. a) An $r z$ cross section of the $\mathrm{LHeC}$ detector in its baseline configuration with the magnet configuration for LR with the solenoid and dipoles placement between the electromagnetic and the hadronic calorimeters. The proton beam, from the right, collides with the electron beam, from the left, at the IP which is surrounded by a central tracker system complemented by large forward and backward tracker telescopes followed by sets of calorimeters. The detector dimensions are $\approx 14 \mathrm{~m}$ in $z$ and a diameter of $\approx 9 \mathrm{~m}$. b) The interaction-region layout. Shown are the beam envelopes of $10 \sigma$ (electrons) [solid blue] or $11 \sigma$ (protons) [solid green], the same envelopes with an additional constant margin of $10 \mathrm{~mm}$ [dashed], the synchroton-radiation fan [orange], the approximate location of the magnet coil between incoming protons and outgoing electron beam [black], and a $1^{\circ}$ line. c) Perspective drawing of the beam pipe and its dimensions. The dimensions consider a $1 \mathrm{~cm}$ safety margin around the synchrotron radiation envelope.

\section{Latest developments}

In the LHeC workshop [5] following the release of the CDR, the CERN management has given a mandate to pursue the required $\mathrm{R} \& \mathrm{D}$ activities and studies for key components of the LR LHeC option, e.g. superconducting RF, and to launch beam dynamics and design studies in the framework of international collaborations. A dedicated collaboration workshop has identified in 2013 a frequency of $801.58 \mathrm{MHz}$ as the optimum choice for the LHeC based on RF power considerations and offering strong synergies with the High-Luminosity-LHC project. An Energy Recovery Linac (ERL) test facility at CERN is now foreseen as a test bed for superconducting RF development, cryogenics, and advanced beam instrumentation, as well as for studies of ERL-specific beam dynamics. The test facility [6] will comprise two linacs, each ultimately consisting of 4 superconducting 5-cell cavities and two return arcs on either side for a final electron energy of about $300 \mathrm{MeV}$. The average beam current should be above $6 \mathrm{~mA}$ to explore the parameter range of the future $\mathrm{LHeC}$.

\section{Conclusions and outlook}

The $\mathrm{LHeC}$ is a project with an ambitious physics program: the recent advances in the available luminosity push the $\mathrm{LHeC}$ project in an even more interesting position as a precision QCD machine and a Higgs factory complementing and enhancing the LHC $p p$ program. A baseline design for the $\mathrm{LHeC}$ detector has been presented. Further developments are ongoing, aiming at a precise simulation of the interaction region first and a framework for a full detector simulation later. The mandate for an ERL test facility at CERN will push the required R\&D and make a roadmap with an LHeC taking data concurrently with the other LHC experiments during the high luminosity program feasible. 


\section{References}

[1] J.L. Abelleira Fernandez et al. [LHeC Study Group Collaboration], A Large Hadron Electron Collider at CERN: Report on the Physics and Design Concepts for Machine and Detector, J. Phys. G 39, 075001 (2012), [arXiv: 1206.2913]

[2] J.L. Abelleira Fernandez et al. [LHeC Study Group], A Large Hadron Electron Collider at CERN, [arXiv:1211.4831]

[3] J.L. Abelleira Fernandez et al. [LHeC Study Group Collaboration], On the Relation of the LHeC and the LHC, [arXiv: 1211.5102]

[4] O. Brüning and M. Klein, The Large Hadron Electron Collider, Mod. Phys. Lett. A 28, 1330011 (2013), [arXiv:1305.2090]

[5] 2012 CERN-ECFA-NuPECC LHeC Workshop, June 2012, Chavannes de Bogis, Switzerland, http://cern.ch/lhec

[6] A. Valloni et al., Strawman optics design for the LHeC ERL Test Facility, Conference Proceedings IPAC13, Shanghai, China, 2013 